\title{
SOME GONFORMALLY SEPARABLE METRICS IN FLAT SPACE AND THEIR APPLICATION TO ACCELERATED COORDINATE FRAMEWORKS IN SPEGIAL RELATIVITY
}

N. W. TAYLOR

(Received 3 March 1967, revised 30 June 1967)

\section{Summary}

If the metric of an $n$-dimensional space is taken in the form $d s^{2}=$ $u^{2} d \tau^{2}+d \sigma^{2}$, where $d \tau^{2}$ and $d \sigma^{2}$ are cartesian metrics of $r$ and $(n-r)$ dimensions, respectively, the various forms of $u$ for flat space are quite simple.

The study of accelerated motions in special relativity by various authors has led to four dimensional metrics of this form. Those in which $u= \pm 1$ at the space origin for all values of time are of particular interest. They are locally cartesian at the accelerated observer, and so the coordinates in the neighbourhood of the observer correspond directly to physical measurements. Hence, such metrics provide convenient means of describing physical conditions experienced by accelerated observers.

If the $\tau$-space contains the time direction and is of one or two dimensions, arbitrary rectilinear motions are allowed.

\section{The metrics}

Consider a metric of the form

$$
\begin{aligned}
& d s^{2} \equiv g_{i j} d x^{i} d x^{j}=\left\{u\left(x^{i}\right)\right\}^{2} d x^{\alpha} d x^{\alpha}+d x^{\mu} d x^{\mu} \\
& \alpha=1, \cdots, r ; \quad \mu=\gamma+1, \cdots, n ; \quad i=1, \cdots, n .
\end{aligned}
$$

This is a special case of the conformally separable metric. More general cases have been used in discussing certain problems in geometry (Yano [10], Wong [9]). However, it is simpler to derive from the beginning the particular results required here than to use formulae from the more general treatments.

The condition for flat space is [8],

$$
\begin{aligned}
R_{i j k l} \equiv & \frac{1}{2}\left(g_{i l, j k}+g_{j k, i l}-g_{i k, j l}-g_{j l, i k}\right) \\
& +g^{m n}([j k, n][i l, m]-[j l, n][i k, m])=0,
\end{aligned}
$$


where, in the terms $g_{i l, j k}$, etc., the comma denotes partial differentiation with respect to the coordinate.

Let $A, B, C$ be different indices, each less than $r$, and let the summation convention be suspended for indices denoted by capital letters. If the metric (1) represents a flat space, then

$$
\begin{aligned}
& R_{A B A B} \equiv u^{3}\left\{(1 / u)_{, A A}+(1 / u)_{, B B}\right\}-u_{, \alpha} u_{, \alpha}-u^{2} u_{, \mu} u_{, \mu}=0 \\
& R_{A B C B} \equiv u^{3}(1 / u)_{, A C}=0 \\
& R_{A \mu A \nu} \equiv-u u_{, \mu \nu}=0 \\
& R_{A B \mu B} \equiv-u^{2}(\log u)_{, A \mu}=0 .
\end{aligned}
$$

All the other components of $R_{i j k l}$ vanish identically. The equations (2) contain the $(r-1)$ equations

$$
(1 / u)_{, A A}=(1 / u)_{, B B}=\cdots .
$$

These equations show that, as far as the value of $r$ is concerned, there are three cases to consider, namely, $r>2, r=2$, and $r=1$.

The CASE $r>2$. From (3)-(6) it follows that

$$
u=\left(2 b_{\mu} x^{\mu}+q\right) /\left\{\left(x^{\alpha}+l^{\alpha}\right)\left(x^{\alpha}+l^{\alpha}\right)+p\right\}
$$

where $b_{\mu}, l^{\alpha}, q$, and $p$ are constants. If this is substituted in any of the equations (2), it is found that $p=b_{\mu} b_{\mu}$. Hence

$$
u=\left(2 b_{\mu} x^{\mu}+q\right) /\left\{\left(x^{\alpha}+l^{\alpha}\right)\left(x^{\alpha}+l^{\alpha}\right)+b_{\mu} b_{\mu}\right\} .
$$

This is valid for any $n-r$, in particular for the case $n-r=0$, where the whole metric is conformal to a cartesian metric. There are then no $\mu$ indices, and so

$$
u=q /\left\{\left(x^{\alpha}+l^{\alpha}\right)\left(x^{\alpha}+l^{\alpha}\right)\right\} .
$$

The CASE $r=2$. There are now no equations of the type (3) and only one of the type (2). It can be written

$$
(\log u)_{, 11}+(\log u)_{, 22}+u_{, \mu} u_{, \mu}=0 .
$$

The other equations are

$$
u_{, \mu \nu}=0
$$

and

$$
(\log u)_{, 1 \mu}=(\log u)_{, 2 \mu}=0 .
$$

From (10) and (11),

$$
u=\left(2 b_{\mu} x^{\mu}+q\right) \exp \left\{\phi\left(x^{\alpha}\right)\right\}
$$


where $b_{\mu}$ and $q$ are constants and $\phi$ is an arbitrary function of $x^{1}$ and $x^{2}$. If this is substituted in (9), it gives

$$
\phi_{, 11}+\phi_{, 22}+4 b_{\mu} b_{\mu} \exp (2 \phi)=0,
$$

a form of Liouville's equation [1]. The solution is

$$
b_{\mu} b_{\mu} \exp (2 \phi)=\frac{-f^{\prime}\left(x^{1}+i x^{2}\right) \cdot g^{\prime}\left(x^{1}-i x^{2}\right)}{\left\{f\left(x^{1}+i x^{2}\right)+g\left(x^{1}-i x^{2}\right)\right\}^{2}}
$$

where $f$ and $g$ are arbitrary functions and the dash denotes ordinary differentiation. Hence, the complete solution is

$$
u^{2}=\frac{-2\left(b_{\mu} x^{\mu}+q\right)^{2} f^{\prime}\left(x^{1}+i x^{2}\right) \cdot g^{\prime}\left(x^{1}-i x^{2}\right)}{b_{\mu} b_{\mu}\left\{f\left(x^{1}+i x^{2}\right)+g\left(x^{1}-i x^{2}\right)\right\}^{2}} .
$$

For the case where the whole metric is conformal to a cartesian metric ( $n=r=2$ ), or for the special solution in the conformally separable system where all the $b_{\mu}$ vanish, the equation for the $\phi$ reduces to Laplace's equation so that

and hence

$$
\phi=F\left(x^{1}+i x^{2}\right)+G\left(x^{1}-i x^{2}\right)
$$

$$
u=f\left(x^{1}+i x^{2}\right) \cdot g\left(x^{1}-i x^{2}\right) .
$$

The CASE $r=1$. For this case, the only set of equations is

$$
u_{, \mu \nu}=0
$$

giving

$$
u=x^{\mu} f_{\mu}\left(x^{1}\right)+b\left(x^{1}\right)
$$

where $f_{\mu}$ and $b$ are arbitrary functions.

\section{Physical conditions on the metrics}

In applications to Relativity theory, $n=4$. For the inertial system in which coordinates correspond directly to physical measurements,

$$
d s^{2}=d x^{i} d x^{i}=d x^{2}+d y^{2}+d z^{2}-c^{2} d t^{2} .
$$

Only those accelerated frameworks will be considered in which the coordinates at the space origin, $O$, of the accelerated observer measure distance and time directly. Hence, $u\left(0,0,0, x^{4}\right)= \pm 1$.

The 4-acceleration of a particle is

$$
F^{i}=d^{2} x^{i} / d s^{2}+\left\{{ }_{j k}^{i}\right\}\left(d x^{j} / d s\right)\left(d x^{k} / d s\right) \text {. }
$$


At the accelerated origin, $O$,

Hence, at $O$,

$$
d x^{1} / d s=d x^{2} / d s=d x^{3} / d s=0, \quad d x^{4} / d s= \pm 1 .
$$

$$
F_{0}^{i}=\left\{{ }_{4}^{i}\right\}_{0} .
$$

The components of acceleration are given by taking $i=1,2,3$. These will vanish in the present case unless $g_{44}=u^{2}$, and so the conformal and conformally separable metrics for accelerated frameworks are

$$
\begin{aligned}
& d s^{2}=(u)^{2}\left\{\left(d x^{1}\right)^{2}+\left(d x^{2}\right)^{2}+\left(d x^{3}\right)^{2}+\left(d x^{4}\right)^{2}\right\} \\
& d s^{2}=\left(d x^{1}\right)^{2}+(u)^{2}\left\{\left(d x^{2}\right)^{2}+\left(d x^{3}\right)^{2}+\left(d x^{4}\right)^{2}\right\} \\
& d s^{2}=\left(d x^{1}\right)^{2}+\left(d x^{2}\right)^{2}+(u)^{2}\left\{\left(d x^{3}\right)^{2}+\left(d x^{4}\right)^{2}\right\} \\
& d s^{2}=\left(d x^{1}\right)^{2}+\left(d x^{2}\right)^{2}+\left(d x^{3}\right)^{2}+(u)^{2}\left(d x^{4}\right)^{2} .
\end{aligned}
$$

With the convention that $x^{4}$ corresponds to time, it is convenient at this stage to number the $\mu$ before the $\alpha$ indices.

The metrics (16) and (17) belong to the type $r>2$. In the case of (16), the equation (8) gives

$$
u^{2}=q /\left\{\left(x^{i}+l^{i}\right)\left(x^{i}+l^{i}\right)\right]
$$

which cannot become \pm 1 at $\left(x^{1}, x^{2}, x^{3}\right)=0$ for all $x^{4}$. This gives the metric used by Page [6], Page and Adams [7], and Gupta [2] in the study of uniformly accelerated motion.

In the case of $(17)$, the $\mu$ index is 1 and the $\alpha$ indices are $(2,3,4)$. The equation (7) then gives

$$
u=\frac{2 b_{1} x^{1}+q}{\left(x^{2}+l^{2}\right)^{2}+\left(x^{3}+l^{3}\right)^{2}+\left(x^{4}+l^{4}\right)^{2}+\left(b_{1}\right)^{2}}
$$

which cannot be \pm 1 at $\left(x^{1}, x^{2}, x^{3}\right)=0$ for all $x^{4}$.

The metric (18) belongs to the type $r=2$. Here, the $\mu$ indices are $(1,2)$ and the $\alpha$ indices $(3,4)$. Hence, from $(12)$,

$$
u=\frac{\left(2 b_{1} x^{1}+2 b_{2} x^{2}+q\right)\left\{-f^{\prime}\left(x^{3}+i x^{4}\right) \cdot g^{\prime}\left(x^{3}-i x^{4}\right)\right\}^{\frac{1}{2}}}{\left\{\left(b_{1}\right)^{2}+\left(b_{2}\right)^{2}\right\}^{\frac{1}{2}}\left\{f\left(x^{3}+i x^{4}\right)+g\left(x^{3}-i x^{4}\right)\right\}}
$$

where the dash denotes ordinary differentiation. So

$$
u\left(0,0,0, x^{4}\right)=\frac{q\left\{-f^{\prime}(\xi) \cdot g^{\prime}(\eta)\right\}^{\frac{1}{2}}}{\left\{\left(b_{1}\right)^{2}+\left(b_{2}\right)^{2}\right\}^{\frac{1}{2}}\{f(\xi)+g(\eta)\}}
$$

where the substitution $\xi=i x^{4}, \eta=-i x^{4}$ is made after the differentiation. Let $h(\xi)=g(-\xi)$. Then, since $d / d \eta=-d / d \xi$,

$$
u\left(0,0,0, x^{4}\right)=\frac{q\left\{f^{\prime}(\xi) \cdot h^{\prime}(\xi)\right\}^{\frac{1}{2}}}{\left.\left\{b_{1}\right)^{2}+\left(b_{2}\right)^{2}\right\}^{\frac{1}{2}}\{f(\xi)+h(\xi)\}} .
$$


Thus, the conditions are satisfied if

$$
q^{2} f^{\prime}(\xi) \cdot h^{\prime}(\xi)=\left\{\left(b_{1}\right)^{2}+\left(b_{2}\right)^{2}\right\}\{f(\xi)+h(\xi)\}^{2} .
$$

Therefore, there is one arbitrary function. If $f$ is chosen, this equation determines the corresponding $g$.

The cases where $b_{1}$ and $b_{2}$ both vanish must be treated separately. From (13),

Then

$$
u=f\left(x^{3}+i x^{4}\right) \cdot g\left(x^{3}-i x^{4}\right) .
$$

$$
u\left(0,0,0, x^{4}\right)=f(\xi) \cdot g(-\xi) .
$$

Hence, $u\left(0,0,0, x^{4}\right)$ can take the values \pm 1 if

In this case,

$$
g(-\xi)= \pm \mathbf{l} / f(\xi)
$$

$$
u= \pm f\left(x^{3}+i x^{4}\right) / f\left(-x^{3}+i x^{4}\right) .
$$

With the metric given by (18) and (20), the components of acceleration of the origin in the $x^{1}$ - and $x^{2}$-directions are constant, $-2 b_{1} / q$ and $-2 b_{2} / q$, respectively. However, like the case (22), it allows an arbitrary acceleration in the $x^{3}$-direction. In the investigation of arbitrary rectilinear motions it is not necesssary to use (20). The simpler form (22) will serve.

For the metric $(19)$, the $\mu$ indices are $(1,2,3)$ and the $\alpha$ index is 4 . The equation (15) gives

$$
u=x^{1} f_{1}\left(x^{4}\right)+x^{2} f_{2}\left(x^{4}\right)+x^{3} f_{3}\left(x^{4}\right)+b\left(x^{4}\right) .
$$

Hence, $u\left(0,0,0, x^{4}\right)= \pm 1$ if $b\left(x^{4}\right)$ is a constant, \pm 1 . So,

$$
u= \pm 1+x^{1} f_{1}\left(x^{4}\right)+x^{2} f_{2}\left(x^{4}\right)+x^{3} f_{3}\left(x_{c}\right) .
$$

In the accelerated framework, the components of acceleration of the origin are $\left(-f_{1},-f_{2},-f_{3}\right)$.

\section{Some transformations}

THE CASE (22). The transformation will now be determined which takes the inertial metric

to

$$
d s^{2}=d x^{\prime 2}+d y^{\prime 2}+d z^{\prime 2}-c^{2} d t^{\prime 2}
$$

where

$$
d s^{2}=d x^{2}+d y^{2}+u^{2}\left(d z^{2}-c^{2} d t^{2}\right)
$$

$$
u= \pm f(z-c t) / f(-z-c t)
$$


Since the motions of interest here are those in the $z$-direction, a transformation will be assumed in the form

The equation

$$
\begin{aligned}
& z^{\prime}=\phi(z, t) \\
& t^{\prime}=\psi(z, t) .
\end{aligned}
$$

for all $z, t$ then gives

$$
d z^{\prime 2}-c^{2} d t^{\prime 2}=u^{2}\left(d z^{2}-c^{2} d t^{2}\right)
$$

and

$$
\phi^{\prime 2}-c^{2} \psi^{\prime 2}=\dot{\psi}^{2}-\phi^{2} / c^{2}=u^{2}
$$

$$
\phi^{\prime} \phi-c^{2} \psi^{\prime} \dot{\psi}=0,
$$

where the dash denotes differentiation with respect to $z$ and the dot differentiation with respect to $t$.

Eliminate $\phi$ from these:

Since $u^{2} \neq 0$,

$$
\left(\phi^{\prime 2}-c^{2} \psi^{\prime 2}\right)\left(\phi^{\prime 2}-\dot{\psi}^{2}\right)=0 .
$$

Then

$$
\phi^{\prime}= \pm \dot{\psi} \text {. }
$$

$$
\dot{\phi}= \pm c^{2} \psi^{\prime}
$$

so that $\phi$ and $\psi$ are each given by the one-dimensional wave equation with velocity $c$. Write the solution for $\phi$ as

Then

$$
\phi=\Phi(z+c t)+F(z-c t)+k .
$$

Hence,

$$
\psi= \pm(1 / c) \Phi(z+c t) \mp(1 / c) F(z-c t)+l .
$$

$$
u^{2}=4 \Phi^{\prime}(z+c t) \cdot F^{\prime}(z-c t) .
$$

$u$ will have the required form, (24), if

$$
F^{\prime}(z-c t)=-1 /\left(4 \Phi^{\prime}(-z+c t)\right) .
$$

(The minus sign is introduced by the change in sign of $z$, while the dash continues to denote differentiation with respect to $z$.) So

$$
F(z-c t)=-\frac{1}{4} \int^{z-c t} d \xi / \Phi^{\prime}(-\xi)
$$

where the dash now denotes differentiation with respect to $\xi$. The required transformation is

$$
\begin{aligned}
& z^{\prime} \equiv \phi=\Phi(z+c t)-\frac{1}{4} \int^{z-c t} d \xi / \Phi^{\prime}(-\xi)+k \\
& t^{\prime} \equiv \psi= \pm(1 / c) \Phi(z+c t) \pm(1 / 4 c) \int^{z-c t} d \xi / \Phi^{\prime}(-\xi)+l
\end{aligned}
$$


If $z^{\prime}=0$ and $t^{\prime}=0$ when $z=0$ and $t=0$, then

$$
\begin{aligned}
z^{\prime} & =\Phi(z-c t)-\Phi(0)-\frac{1}{4} \int_{0}^{z-c t} d \xi / \Phi^{\prime}(-\xi) \\
t^{\prime} & = \pm(1 / c) \Phi(z+c t) \mp(1 / c) \Phi(0) \pm(1 / 4 c) \int_{0}^{z-c t} d \xi / \Phi^{\prime}(-\xi) .
\end{aligned}
$$

Different motions are given by taking different functions, $\Phi(\xi)$. Lass [3] treats the case of uniformly accelerated motion, for which

Then

$$
\Phi(\xi)=\left(c^{2} / 2 g\right) \exp \left(g \xi / c^{2}\right) .
$$

and

$$
u^{2} \equiv-\Phi^{\prime}(z-c t) / \Phi^{\prime}(-z+c t)=\exp \left(2 g z / c^{2}\right)
$$

$$
\begin{aligned}
z^{\prime} & =\left(c^{2} / g\right) \exp \left(g z / c^{2}\right) \cdot[\cosh (g t / c)-1] \\
t^{\prime} & =(c / g) \exp \left(g z / c^{2}\right) \cdot \sinh (g t / c) .
\end{aligned}
$$

THE CASE (23). The transformation which takes the inertial metric

$$
d s^{2}=d x^{\prime 2}+d y^{\prime 2}+d z^{\prime 2}-c^{2} d t^{\prime 2}
$$

to

where

$$
d s^{2}=d x^{2}+d y^{2}+d z^{2}-c^{2} u^{2} d t^{2}
$$

$$
u= \pm 1+x f_{1}(t)+y f_{2}(t)+z f_{3}(t)
$$

is the transformation to an accelerated rigid system of reference (Møller [5], p. 253).

A special case of such a motion occurs when the space origin has an arbitrary acceleration in the $z$-direction, and the space axes remain parallel. The transformation in the form given by Marsh [4] is

$$
\begin{aligned}
z^{\prime} & =z\left[1+\dot{\Psi}^{2} / c^{2}\right]^{\frac{1}{2}}+\Psi \\
t^{\prime} & =z \dot{\Psi} / c^{2}+\int_{0}^{t}\left[1+\dot{\Psi}^{2} / c^{2}\right]^{\frac{1}{2}} d t
\end{aligned}
$$

where $\Psi$ is a function of $t$ only. Then

$$
u= \pm\left(1+z \ddot{\Psi} /\left(c^{2}\left[1+\dot{\Psi}^{2} / c^{2}\right]^{\frac{1}{2}}\right)\right) .
$$

\section{References}

[1] A. R. Forsyth, Theory of Differential Equations. Vols. 5 and 6, Partial Differential Equations. Dover, New York, (1959).

[2] S. N. Gupta, "Conformal transformations and space travel". Science 134 (1961), 13601361.

[3] H. Lass, "Accelerating frames of reference and the clock paradox". Am. J. Phys. 31 (1963), $274-276$.

[4] L. McL. Marsh, "Relativistic accelerated systems". Am. J. Phys. 33 (1965), 934-938.

[5] C. Moller, The Theory of Relativity. Oxford (1952). 
[6] L. Page, "A new relativity". Phys. Rev. 49 (1936) $254-268$.

[7] L. Page and N. I. Adams, Electrodynamics. Dover, New York (1965).

[8] I. S. Sokolnikoff, Tensor Analysis. Theory and Applications to Geometry and Mechanics of Continua. John Wiley \& Sons, Inc., New York (1964).

[9] Y. C. Wong, "Some Einstein spaces with conformally separable fundamental tensors". Trans. Am. Math. Soc. 53 (1943), 157-194.

[10] K. Yano, "Conformally separable quadratic differential forms". Proc. Imp. Acad. Tokyo 16 (1940), 83-86.

Department of Mathematics

University of New England

Armidale

New South Wales 